\title{
Spray Dispersion Measurements of a Sprinkler Array
}

\author{
E.D. Link, S.J. Jordan, T.M. Myers, P.B. Sunderland, and A.W. Marshall*
}

Department of Fire Protection Engineering, University of Maryland, 4356 Stadium Dr., College Park, MD 20742, USA

Corresponding author email: awmarsh@umd.edu

Colloquium: Fire Research

Paper Length: 5262 words, Method 1

Main Text: 3324

References: 297

Figure 1: 164

Figure 2: 354

Figure 3: 373

Figure 4: 312

Figure 5: 208

Figure 6: 254

(C) 2016. This manuscript version is made available under the Elsevier user license 


\begin{abstract}
A careful measurement methodology has been developed for highly resolved spray dispersion measurements. These measurements are used to establish spatial resolution requirements for evaluation of sprinkler spray patterns and to provide a comprehensive data set for computer model validation. For this study, four nozzles were arranged in a square configuration typical of fire sprinkler system installations. Volume flux of water delivered to the floor was measured $1.5 \mathrm{~m}$ below the array at locations on a $50 \mathrm{~mm}$ grid. The detailed measurements accurately portray the spatial variations existing in the spray and indicate that each sprinkler contributes a unique spray pattern. The total mean volume flux was $5.9 \mathrm{~mm} / \mathrm{min}$, with local measurements ranging from $25 \%$ to nearly $400 \%$ of the mean value. A spatial averaging analysis suggests that at least 25 measurements should be taken across the reach (i.e. extent) of the spray to resolve gradients in the spray pattern. Fewer measurements provide limited insight beyond the overall mean flux (due to resolution errors), while more measurements become increasingly expensive and less accurate (due to repeatability errors). To quantify the significant spray pattern variations, a meticulous measurement and alignment routine is described to ensure results are sufficiently comprehensive, detailed, and accurate for use in computational model validation.
\end{abstract}

Keywords: fire, suppression, delivered density, volume flux, validation 


\section{Introduction}

Water-based fire suppression methods are widely used for their effectiveness, simplicity, and availability. Automatic fire sprinkler installations in particular represent a predominant fire suppression strategy, and have been established as a reliable and effective method to reduce property and life safety losses. There are dozens of sprinkler designs commercially available, each producing a unique spray dispersion pattern. Performance is often characterized through evaluation of the spray pattern in terms of a water volume flux distribution on wetted surfaces. These spray patterns govern fire suppression efficacy. In fact, sprinklers are designed with a specific spray pattern depending on the application. For example, ESFR type sprinklers with a downward biased spray pattern for plume penetration are used in warehouse storage applications [1] while residential type sprinklers with a wide spray pattern for wall protection are used in residential occupancies. These sprinklers are approved for fire protection use according to their performance in various standard tests, which include spray pattern evaluation.

Early measurements of sprinkler dispersion were conducted by Beyler [2] to investigate the influence of several variables on the volume flux distribution, including sprinkler installation orientation, flow rate, and frame arm position. Water was collected in $0.3 \mathrm{~m}$ square bins arranged on the floor in either radial or rectangular gridded patterns. For measurement of multiple sprinklers, the rectangular grid provided a simpler coordinate system and more complete floor coverage. This early study introduced a spatial volume flux measurement method, but only limited improvements have been implemented in subsequent studies. Due to experimental uncertainties, large collection bins, and lack of sprinkler characterization, the data set is limited and inappropriate for model validation.

FM Global has provided many of the large-scale spray dispersion studies available in the literature. These studies focused on quantifying spray patterns through measurement of volume flux distribution, also termed local delivered density, both under quiescent conditions and in the presence of a fire $[1,3,4]$. Local density measurements were reported by Yao, also using a series of $0.3 \mathrm{~m}$ square bins, arranged in a radial line and swept around the centerline to provide an azimuthal average 
measurement of the spray distribution area at various heights below the sprinkler [3]. Subsequent density measurements were taken in the presence of a fire in rack storage applications and used a grid of 16 square pans approximately $0.5 \mathrm{~m}$ in width [4]. Measurements using a similar radial sampling technique were performed by Prahl and Wendt [5] using $0.25 \mathrm{~m}$ square bins, although with an idealized axisymmetric sprinkler. A more spatially-resolved measurement technique was used by Chow and Wong, who implemented a rectangular grid of $120,0.25 \mathrm{~m}$ square collection bins to measure the spray penetration ratio in the presence of a crib fire [6]. Their findings focused on the penetration ratio and the influence of the fire on spray dispersion rather than on spray measurement details. Even from visual observation of an operating sprinkler, initial spray non-uniformities and their far-field propagation are apparent, motivating the need for detailed spray measurements. More recent studies have quantified spatial variations in spray characteristics and volume flux distribution to support detailed analysis of fire suppression performance [7-10].

As use cases for computational modeling in fire protection design become increasingly ambitious, validation of spray dispersion simulations has emerged as a critical research activity for the development of methods and models to predict water-based fire suppression performance. Missing from previous spray dispersion efforts is a complete data set resolving the details of spray dispersion (from initialization to delivery) for comparison with simulations. Several questions remain regarding sprinkler spray dispersion, such as the extent of spray pattern non-uniformity and best practices for accurate measurement of spatial variations in the spray, particularly for the purpose of model validation. The current industry standard for sprinkler tests is reflected in a number of Underwriters Laboratories standards [11,12], using large $0.3 \mathrm{~m}$ collection pans and evaluating spray dispersion with pass/fail criteria based on a maximum allowable number of pans having a volume flux below a critical threshold value.

The present experimental study investigates the far-field non-uniformity of a sprinkler spray using an array of sprinklers arranged in a square grid, characteristic of typical sprinkler installations. 
Detailed measurements of volume flux at the floor, at over 2500 locations, were obtained to evaluate the spray pattern, as well as establish experimental methods and guidelines for accurately capturing the variations in the spray. Careful experimental design and measurements provide a data set that can be used for future CFD validation. To complete the data set for model validation, near-field volume fluxspray measurements are also presented, providing critical information for spray initialization. Nearfield measurements including the important spatially-resolved volume flux distribution, drop size distribution and velocity measurements are critical for spray initialization [13]. Specifics of the near

field initial spray measurements are beyond the scope of this paper, and the reader is directed to work by Jordan [1413] for details related to this measurement. It is acknowledged that the spray dispersion and fire suppression problem is heavily dependent on flows induced by the fire (e.g. fire plumes), and future work is planned to investigate these effects upon completion of this study in the simplified quiescent configuration.

\section{Experimental Approach}

\subsection{Facility Description}

In the current study, four sprinklers were arranged in a square grid configuration, with spacing of $2.65 \mathrm{~m}$, shown in Fig. 1. All sprinklers were Tyco D3 spray nozzles with a k-factor of 33.1 LPM/bar ${ }^{1 / 2}$, operating at 1.38 bar and flowing a combined total of $155 \mathrm{~L} / \mathrm{min}$. The measured spray pattern for each individual sprinkler varied slightly, so all were given identifying numbers and the angular position of each was carefully aligned.




Figure 1. Drawing of the experimental facility showing the arrangement of the sprinklers and the water collection tubes. The circled numbers identify each sprinkler.

Water was supplied to the system by an underground storage tank and pump. Flow rate was regulated with a valve coupled to an electronic controller monitoring the total pressure just upstream of each sprinkler. Pressure measurements were acquired with an Omega PX302 pressure transducer, with range $0-13.8$ bar and accuracy $0.25 \%$ BFSL.

\subsection{Diagnostics and Measurements}

A series of collection tubes, $52 \mathrm{~mm}$ in diameter and depicted in Fig. 1, measured the volume flux, $\dot{V}^{\prime \prime}$, of water to a plane $1.5 \mathrm{~m}$ beneath the sprinkler deflectors. Volume flux measurements beneath the full array were obtained over a series of tests. The test procedure begins with covered collection tubes while the water flow-rate stabilizes at a steady-state operating pressure. At this point the $\underline{\text { tubes are uncovered and collect water for } 10 \text { minutes before being covered once again. After the water }}$ flow is terminated, the water pressure head in each tube is measured. In each test, the tubes were manually positioned to obtain measurements every $50 \mathrm{~mm}$, such that the round collection tubes cover $85 \%$ of the total gridded area. Effects from the lip of the collection tubes were determined to have no material impact on the flux accuracy based upon measurements over a range of collection configurations. A Setra 209 pressure transducer, range 0-0.07 bar and accuracy $\pm 0.25 \%$ FS, measured the pressure head of water accumulated in each tube. With the assumption that all water reaching the open area of the tube is collected, the volume flux - -was determined by $\dot{V}^{\prime \prime}=\left(A_{t} \Delta h\right) /\left(A_{o} t\right)$, where Using the area of the collection tube, $A_{t}$ is the area of the collection tube, the area of the tube opening, $A_{o}$ is the area of the tube opening, the collection time duration, $t$ is the collection time duration, the flum, $\dot{V}^{\mu}=\left(A_{t} \Delta h\right) /\left(A_{\theta} t\right)$. 
Near-field spray measurements were also performed for each individual sprinkler using the Spatially-Resolved Spray Scanning System (4S), a unique facility capable of measuring the volume flux, drop size, and drop velocity of the spray on a near-field spherical surface surrounding the nozzle corresponding to the spray formation region, providing detailed data for model input and initialization [1413]. By measuring each sprinkler independently, sprinkler-to-sprinkler variations are accurately documented and the relation of spray patterns in the near field can be linked to far field dispersion.

\subsection{Analytical Methodology}

Measurements of sprinkler arrays show strong spatial variations in far-field volume flux. One objective of the present work is to evaluate the spatial measurement resolution necessary to capture these variations. Error relative to the true spray pattern will be introduced if the measurement resolution is inadequate. It is also recognized that a measurement repeatability error is present due to variables including positioning of the collection tubes, water pressure variations, temperature, room airflow, or other variations in spray pattern from day to day. Rigorous alignment and flow control procedures were implemented to reduce the effects of such variables. Repeatability error was quantified through analysis of the standard error of repeat measurements at each location.

Resolution error was quantified by evaluating local differences in volume flux between the measured high-resolution data set and several low-resolution data sets constructed by averaging neighboring data points. The characteristic grid cell size, $d x$, describes the coarseness of the spatial grid. The error associated with the spatial averaging was determined by comparing the interpolated lowresolution data sets to the high-resolution reference case. The error, $\epsilon$, was calculated with a normalized standard deviation by

$$
\epsilon=\frac{\left[N \sum_{i=1}^{N}\left(H R_{i}-L R_{i}\right)^{2}\right]^{1 / 2}}{\sum_{i=1}^{N} H R_{i}}
$$

where $H R_{i}$ is the individual high-resolution measurement at each location $i$, and $L R_{i}$ is the value of the low-resolution data at each measurement location, and $N$ is the total number of measurement locations. 
Of more specific concern to regulatory testing bodies is the accurate identification of the lowflux areas of the far-field spray. These low-flux areas are associated with insufficient wetting, the primary suppression mode used in sprinkler protection. These 'dry' regions are identified through a threshold criterion applied to the volume flux measurement, and can be quantified by simply counting the number of discrete bins with insufficient volume flux. However, if the collection bins are too large, errors caused by the discretization will contaminate the evaluation of the low flux regions. These errors can be reduced with better bin resolution or through a simple interpolation scheme.

Resolution is determined by the number of measured points in a length scale of interest, not explicitly by the spacing of the points. Therefore, resolution must be discussed relative to a characteristic length rather than in terms of absolute length. For a sprinkler spray, one characteristic length scale is the spray reach, $R$, or distance from the sprinkler head that encompasses all of the wetted area. The simplest estimation of spray reach assumes the drops follow projectile behavior with no drag $[5,1514]$. This model, while simple, may significantly over predict the spray reach by omitting drag and viscous influences. A more accurate approximation is to use the numerical solution of the differential equation for droplet momentum, incorporating the effects of drag. The most accurate method, used in the current analysis, is the direct measurement of spray reach by measurement of the spray pattern and determination of the wetted area.

\section{Results and Discussion}

\subsection{Initial Spray Measurements}

Each individual sprinkler was completely characterized (i.e. spatially resolved volume flux, drop size, and velocity measurements) using the 4S facility [1413] to provide data for CFD initial conditions.

Detailed spatially-resolved spray measurements for these sprinklers reveal that an overall drop size distribution characterized by a Log-Normal Rosin-Rammler distribution [9] with volume median drop $\underline{\text { diameter }} d_{v 50}=0 . \underline{5779} \mathrm{~mm}$, drop size distribution parameter, $\Gamma=2 . \underline{57}$, and a characteristic radial velocity of $6.9 \mathrm{~m} / \mathrm{s}$, are suitable for describing the spray along with the volume flux distributions 
provided in Fig. 2. Sprinklers were marked with a consistent datum for installation and measurement to identify the angular position. The Tyco D3 spray nozzle deflector, consisting of a $26 \mathrm{~mm}$ diameter disc with 12 rotationally symmetric slot/tine pairs each $30^{\circ}$, is shown in Fig. $2 \mathrm{a}$, with the reference positions of $90^{\circ}$ and $270^{\circ}$ identified by frame arms. Sprinklers were oriented as shown in Fig. 2a with $90^{\circ}$ in the positive y-direction. Measurements were performed at a radius of $0.4 \mathrm{~m}$ from the deflector as indicated in Fig. $2 \mathrm{~b}$, for a complete $360^{\circ}$ revolution around the centerline and up to $10^{\circ}$ above horizontal. The near-field volume flux measurements of the relevant quadrant, shaded in Fig. 2b, are shown in Fig. 2c for each individual sprinkler. Each quarter hemisphere represents the quadrant of the spray directed into the array. The circled numbers correspond to the location of the sprinklers identified in Fig. 1. It should be noted that these measurements reveal that even sprinklers of the same design produce different spray patterns at identical injection pressures.

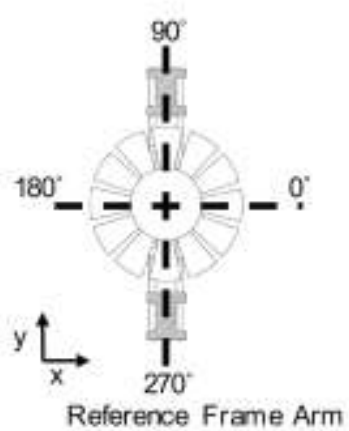

(a)



(b)

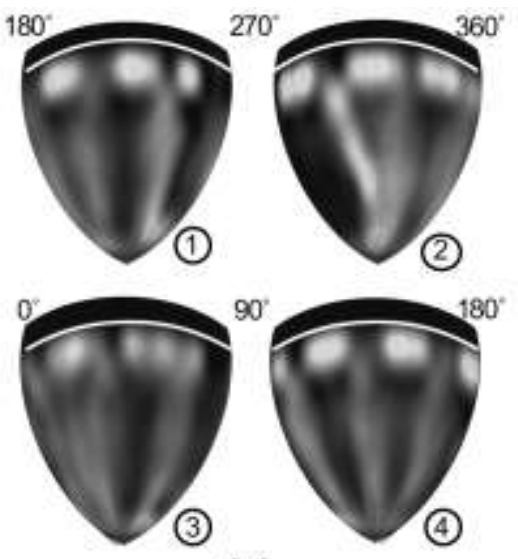

(c)

Figure 2. Diagrams showing the sprinkler geometry and identification of alignment reference points; (a) Top-down view of sprinkler deflector and frame arms, (b) orientation of the initialization sphere, (c) initial spray volume flux measurements of each sphere quadrant.

\subsection{Far-field Volume Flux Measurements}

The set of 2970 far-field volume flux measurements from the array is shown in Fig. 3a. The individual sprinklers are also identified in Fig. 3a, along with the orientation of the frame arms along the y-axis. Non-uniformities in the dispersion are evident by the radial finger-like structures from the 
centerline of each sprinkler that appear to correlate with the specific deflector geometry and frame arms. These non-uniformities are quantified in Fig. 3b, showing the probability distribution of the individual flux measurements. Volume flux measurements ranged from 1.5 to $21.0 \mathrm{~mm} / \mathrm{min}$, with a mean of 5.9 $\mathrm{mm} / \mathrm{min}$ and a standard deviation of $2.0 \mathrm{~mm} / \mathrm{min}$. The mean flux compares favorably with the common metric described by the flow rate divided by the protected area of $5.6 \mathrm{~mm} / \mathrm{min}$.

(a)

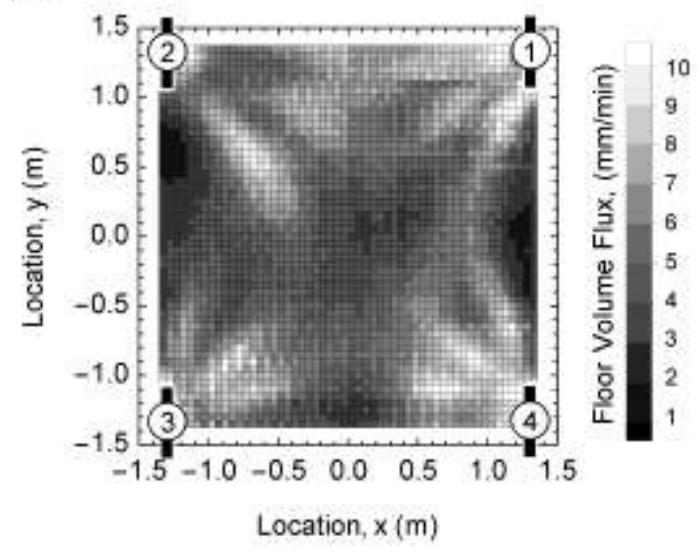

(b)

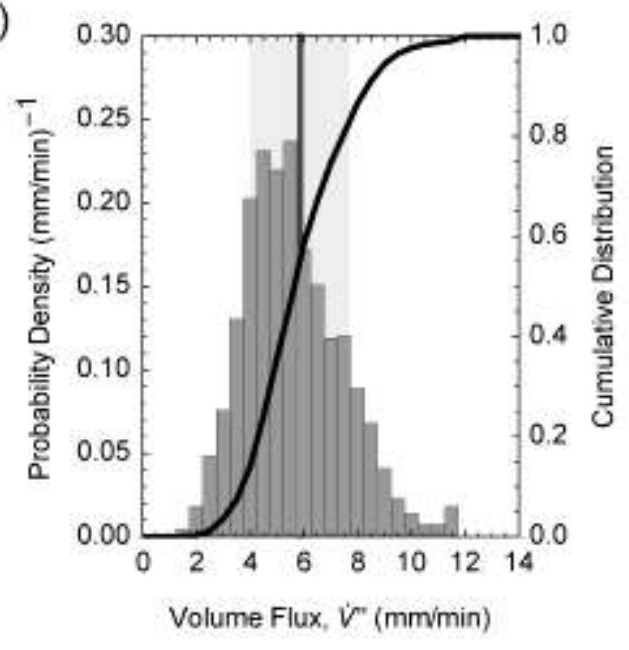

Figure 3. (a) Volume flux measurements on a $0.05 \mathrm{~m}$ grid at 2970 locations on the floor. Each sprinkler is identified at its location. (b) Probability density and cumulative distribution of volume flux measurements.

\subsection{Repeatability and Resolution Analysis}

Calibration of the pressure transducer measurement determined the measured height to be linear within $0.26 \%$ of the calibration at $95 \%$ confidence. This component of the measurement contributes very little to the overall measurement error due to the high accuracy of the pressure transducer. The larger sources of error are presented by other system variables described in Section 2.3 (e.g. exact collection tube placement), and are quantified in the repeatability error. The repeatability error of the 2970 individual volume flux measurements was determined to be $7.5 \%$ using a $95 \%$ confidence interval.

Resolution error is introduced by using large collection bins, which cannot capture the details of the spray pattern. Figure 4 shows the results of spatial averaging at several different length scales, providing a qualitative comparison of the effect of measurement resolution. Fig. 4a shows the high- 
resolution $0.05 \mathrm{~m}$ grid measurements, Fig. $4 \mathrm{~b}$ the same data averaged over an intermediate $0.15 \mathrm{~m}$ grid, and Fig. $4 \mathrm{c}$ the data averaged over a $0.30 \mathrm{~m}$ grid, the bin size of most previous research and the UL test standard. As the bin size increases, the flux in each area approaches the mean flux of the entire spray. Through this binning, spray details are smoothed out, gradients are obscured, and the extremes of flux measurement are lost. This effect is evident in the $0.30 \mathrm{~m}$ bins, where only the large scale trends are resolved and the extent of the low flux regions, identified as locations with volume flux less than one standard deviation below the mean volume flux, are misrepresented.

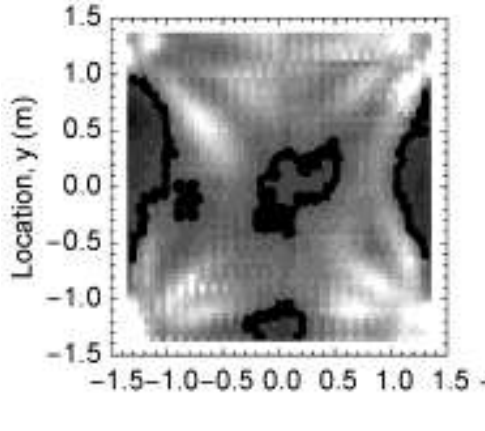

(a)

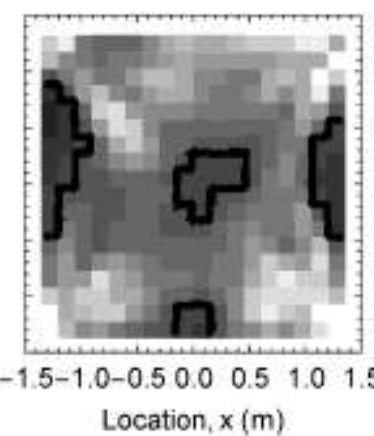

(b)

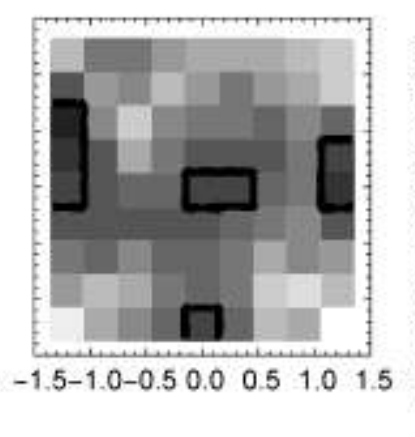

(c)

Figure 4. Spatial averaging of the data using grid sizes (a) $0.05 \mathrm{~m}$, (b) $0.15 \mathrm{~m}$, (c) $0.30 \mathrm{~m}$. Contour lines outline the bins identified as low flux locations with volume flux less than 1 standard deviation (31\%) below the mean.

Fig. 5 shows errors due to both the spatial resolution of the measurement locations and error associated with measurement repeatability. The errors are compared for varying measurement resolution, $d x / R$, where $d x$ is the collection bin length and $R$ is the measured spray reach as described in Section 2.3. For the current experimental conditions $R=3.1 \mathrm{~m}$, determined by direct measurement of the spray reach. 


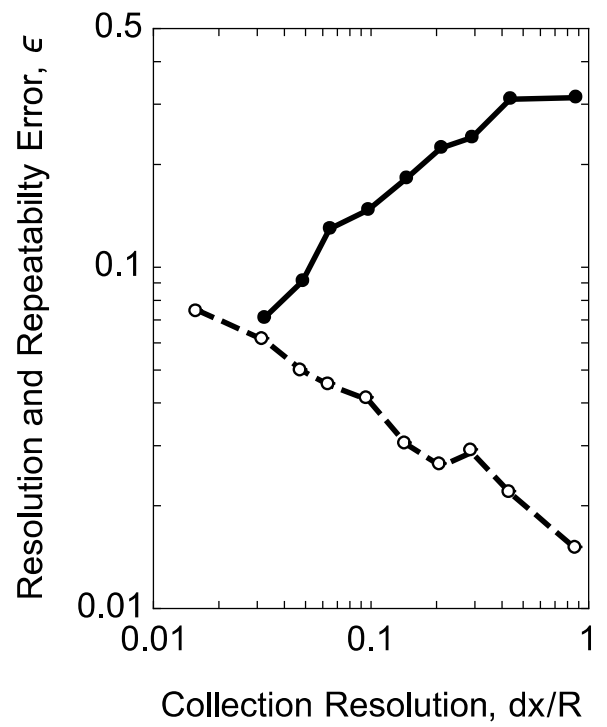

Figure 5. Resolution error (solid) and repeatability error (dashed) plotted as a function of collection resolution.

The solid line in Fig. 5 indicates the resolution error as quantified by Eq. (1). As expected, resolution error increases as individual collection tube area increases. The dashed line shows the repeatability error calculated for each resolution. Repeatability error improves from $7.5 \%$ at the highest resolution to $1.5 \%$ for the lowest resolution. As the spatial detail of the measurement is reduced, local mean flux may be more accurately measured. A comparison between the two errors highlights the compromise between spatial resolution and measurement repeatability. Assuming the two error types are non-additive, $\mathrm{t}$ The lowest total error occurs at the near-intersection of the each curvelines, where the error from both resolution and repeatability are low and of comparable magnitude. To the left of this intersection-point the measurement is of a high resolution but introduces increased repeatability error. To the right, at low resolutions, the improved repeatability comes at a significant increase in resolution errors, providing a good measurement of spatially filtered flux, which has limited utility. Further discussion of repeatability and resolution error on local volume flux measurements is provided after the following discussion of resolution errors on the integral measurement of low flux area. 
Resolution errors in the integral measurement of the low flux areas become apparent from simply totaling the number of low flux bins. Figure 4 shows contours of the low flux bins at several resolutions. The identification of low flux regions is less accurate at low resolutions because the bins are too large to resolve the variations in the spray pattern. The points in Fig. 6 plot the total area covered by the low flux bins for two threshold values. The solid symbols correspond to a low flux threshold $25 \%$ below the mean volume flux, while the open symbols correspond to a threshold one standard deviation (31\%) below the mean volume flux. Scatter in the trends indicates errors associated with the bin discretization, and contributes to additional error in resolving the low flux area. The smooth lines in Fig. 6 show how an interpolation of the binned volume flux alleviates discretization errors and improves the resolution of the low flux area.

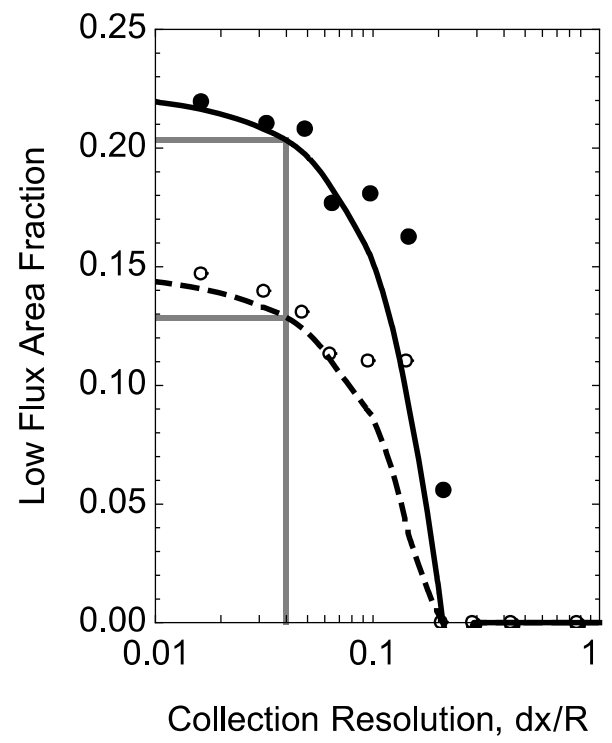

Figure 6. 'Dry' regions; fraction of total measurement area with subcritical flux as a function of collection resolution. Area is determined by number of bins (points). Interpolation between bins reduces discretization errors (dark lines). Low flux thresholds are $25 \%$ (solid) and $31 \%$ (empty) of the mean volume flux. Nominal resolution $d x / R=0.04$ corresponds to resolved area reduction of $90 \%$ (gray lines). 
The results in Fig. 5 show that the resolution errors are comparable to the repeatability error and less than $10 \%$ at a resolution of $d x / R=0.04$, or at least 25 measurements across the spray reach. At $d x / R=0.04$, the repeatability is slightly improved from the best resolution $(d x / R=0.015)$, and the resolution error has not increased beyond $10 \%$, indicating a tolerable increase in error with reduction in measurement resolution to this level. The corresponding binned volume flux measurements for $d x / R=0.04$ are highlighted in Fig. $4 \mathrm{~b}$ along with the outlined low flux area allowing visualization of spray pattern structures at this minimum suggested resolution. Additionally, Fig. 6 reveals that the low flux area can be resolved to within $90 \%$ of the best-resolved area at a resolution of $d x / R=0 . \underline{0} 4$ indicated by the gray lines. The qualitative visual agreement, coupled with quantitative estimates of minimal error in local flux and integral low flux area measurement suggest that the measurement resolution criterion of $d x / R<0.04$ is suitable to accurately resolve volume flux variations. While the spray reach, $R$, is used here to provide a general framework for discussion of resolution, additional variables such as injection pressure and deflector geometry, may influence the gradients present in the dispersed spray.

\section{Conclusions}

A set of highly resolved volume flux measurements in a sprinkler array have been collected to provide insight into the measurement of sprinkler spray pattern non-uniformities and to support CFD model validation. The measured volume flux variations of the initial spray (in the near-field) and near the floor (far-field) correlate with sprinkler geometry details (e.g. frame arm positioning and slot/tine patterns). However, the measured initial volume flux is unique for each sprinkler, despite sprinkler model and injection condition uniformity. Detailed characterization of sprinkler injection conditions, including volume flux distribution, drop size, and velocity, is therefore essential for the purpose of model validation. While measuring volume flux with large area collection bins reduces the time required to characterize the spray, such a method hides the gradients in far-field spray volume flux and obscures low-flux areas (i.e. dry regions) that may influence suppression efficacy. Analysis of the ultrafine grid measurement data provides guidance for the measurement resolution required to resolve spray 
pattern details. Both qualitative visual inspection of the volume flux and a quantitative error assessment suggest that a resolution of $d x / R=0.04$ is required, corresponding to at least 25 data points across the spray reach. At this resolution, local error in spray pattern is not dramatically influenced by the measurement grid size, comparisons of the integral low flux areas are favorable, and the resolution is compatible with current CFD capabilities.

\section{Acknowledgements}

This research was funded by the U.S. National Science Foundation (Award \#1236788), FM Global, and United Technologies Research Center. Appreciation is extended to R. Bentley for his assistance with data collection, as well as; A. Trouve; and J. White at the University of Maryland, and $\mathrm{H}$.

Z. Yu of FM Global, for their insight and contributions. 


\section{References}

[1] C. Yao, Fire Safety Science-Proceedings of the Fifth International Symposium (1997) 93-110.

[2] C.L. Beyler, Effect of Selected Variables on the Distribution of Water from Automatic Sprinklers, Report No. NBS-GCR-77-105, U.S. National Bureau of Standards, 1977.

[3] C. Yao, Applications of Sprinkler Technology_-Early Suppression of High-Challenge Fires with Fast-Response Sprinkler, Proceedings of the Symposium on Application of Fire Science to Fire Engineering, ASTM STP882 (1985) 354-376.

[4] C. Yao, Fire Safety J. 14 (1988) 65-73.

[5] J.M. Prahl, B. Wendt, Fire Safety J. 14 (1988) 101-111.

[6] W.K. Chow, V.M.K. Wong, Proc. First Asia-Oceania Symp. on Fire Science and Technology (1992) $467-474$.

[7] D.T. Sheppard, Spray Characteristics of Fire Sprinklers, Report No. NIST GCR 02-838, National Institute of Standards and Technology, 2002.

[8] A.W. Marshall, Fire Safety Science-Proceedings of the Tenth International Symposium (2011) 6176.

[9] N. Ren, H.R. Baum, A.W. Marshall, Proc. Combust. Inst. 33 (2011) 2547-2554.

[10] X. Zhou, H-Z. Yu, Fire Safety J. 46 (2011) 140-150.

[11] UL 199: Standard for Safety-Automatic Sprinklers for Fire Protection Service, Underwriters Laboratories, (2013).

[12] UL 1626: Standard for Safety-Residential Sprinklers for Fire-Protection Service, Underwriters Laboratories, (2012).

[13] T. Myers, A.W. Marshall, Fire Safety J. (2016) Accepted for publication.

[1413] S. Jordan, A.W. Marshall, Fire Tech. (to be submitted 2016).

[1514] A. Blum, Discharge Characteristics of Canonical Sprinkler Sprays, MS thesis, University of Maryland, College Park, MD, USA, 2007. 


\section{Figure Captions}

Figure 1. Drawing of the experimental facility showing the arrangement of the sprinklers and the water collection tubes. The circled numbers identify each sprinkler.

Figure 2. Diagrams showing the sprinkler geometry and identification of alignment reference points; (a) Top-down view of sprinkler deflector and frame arms, (b) orientation of the initialization sphere, (c) initial spray volume flux measurements of each sphere quadrant.

Figure 3. (a) Volume flux measurements on a $0.05 \mathrm{~m}$ grid at 2970 locations on the floor. Each sprinkler is identified at its location. (b) Probability density and cumulative distribution of volume flux measurements.

Figure 4. Spatial averaging of the data using grid sizes (a) $0.05 \mathrm{~m}$, (b) $0.15 \mathrm{~m}$, (c) $0.30 \mathrm{~m}$. Contour lines outline the bins identified as low flux locations with volume flux less than 1 standard deviation (31\%) below the mean.

Figure 5. Resolution error (solid) and repeatability error (dashed) plotted as a function of collection resolution.

Figure 6. 'Dry' regions; fraction of total measurement area with sub-critical flux as a function of collection resolution. Area is determined by number of bins (points). Interpolation between bins reduces discretization errors (dark lines). Low flux thresholds are $25 \%$ (solid) and $31 \%$ (empty) of the mean volume flux. Nominal resolution $d x / R=0.04$ corresponds to resolved area reduction of $90 \%$ (gray lines). 